\title{
Fine-to-Coarse Multiscale Permutation Entropy for Rolling Bearing Fault Diagnosis
}

\author{
Zhiqiang Huo ${ }^{1,2}$, Yu Zhang ${ }^{1}$, Lei Shu ${ }^{2,1 *}$ \\ ${ }^{1}$ School of Engineering, University of Lincoln, Lincoln, UK \\ ${ }^{2}$ Guangdong Provincial Key Laboratory on Petrochemical Equipment Fault Diagnosis, \\ Guangdong University of Petrochemical Technology, Maoming, China \\ Email: \{zhuo, yzhang, lshu\}@lincoln.ac.uk
}

\begin{abstract}
Multiscale Permutation Entropy (MPE) has been applied as a non-linear measure for estimating the complexity of time series. Nevertheless, the coarse-grained procedure in MPE only takes low-frequency information into account. To overcome this shortcoming, in this paper, a new entropy measure, named Fine-to-Coarse Multiscale Permutation Entropy (F2CMPE), is proposed to provide stable and reliable results by offering both low-frequency and high-frequency information. Firstly, the F2C signals are created based on the reconstruction of selected wavelet coefficients using wavelet packet decomposition. Then, permutation entropy is used to estimate the complexity and dynamic change of the F2C signals. Experimental analysis is carried out to investigate and compare the performance of the proposed F2CMPE with that of the MPE. Results indicate that the proposed method can give consistent and stable entropy measure for rolling bearing fault diagnosis.

Index Terms-Fault Diagnosis, Rolling Bearing, Fine-to-Coarse
\end{abstract} Procedure, Wavelet Packet Decomposition, Permutation Entropy

\section{INTRODUCTION}

Rolling bearings have been widely used in industrial mechanical systems. They are typically operated under harsh environment and are inevitably subjected to incipient defects which could lead to unexpected energy waste and performance degradation. Hence, fault diagnosis of incipient bearing failures not only improves the safety and reliability of the whole system but also avoids financial losses caused by breakdowns. For this purpose, a lot of efforts have been put into the field of monitoring and diagnosing incipient bearing faults using statistical measurements aiming at discriminating fault characteristics hidden in complex signals as early as possible, such as time-domain features and frequency-domain features. Nevertheless, both of time-domain and frequency-domain features are most suitable for linear and stationary signals. As a result, traditional linear methods may not efficiently detect the dynamic change of non-stationary vibration signals [1].

In recent decades, continuing advances in entropy-based measurements have provided the possibility of estimating the complexity and irregularity in non-linear and non-stationary signals, which are suitable for analyzing industrial complex signals. The commonly used entropy-based measurements include single-scale entropy approaches (e.g., Sample Entropy

Corresponding author: Lei Shu (email: 1shu@lincoln.ac.uk)
(SE) and Permutation Entropy (PE)) and multiple-scale entropy approaches (e.g., Multiscale Entropy (MSE)) [2], [3]. In 2002, Costa [4] proposed a method called MSE, which enables to separate regular and pathological groups by using coarsegrained signals obtained from the original signal. The major ideas behind the concept of MSE can be simply concluded as two stages: 1) obtain coarse-grained time series representing the dynamics from the original time series based on the coarsegrained procedure; 2) estimate the complexity and irregularity of coarse-grained time series using SE. Based on this, several multiscale-based entropy measurements were later proposed, such as multiscale fuzzy entropy, and Multiscale Permutation Entropy (MPE) [5]. Nevertheless, it was reported that the MPE still has some shortcomings to be improved [6], [7]. Indeed, the fundamental theory behind the coarse-grained procedure is a linear smoothing of the original time series. As a result, with a large scale factor, it generates signals with reduced length, which is not suitable for short-length time series. Moreover, high-frequency information is ignored in coarse-grained time series. Obviously, this may not effectively detect and diagnose failures in rotating components especially when coherent fault symptoms are hidden in high-frequency components.

In this paper, a novel entropy measure, named Fine-toCoarse Multiscale Permutation Entropy (F2CMPE), is put forward to give a reliable entropy measure. Firstly, Wavelet Packet Decomposition (WPD) is used to produce the reconstructed signals from selected coefficients. After that, the F2C procedure is used to generate $\mathrm{F} 2 \mathrm{C}$ signals which have different frequency range containing low and high-frequency information. Then, PE is used to measure the complexity of F2C signals. The main contributions in this paper are concluded as follows:

- A novel measure, named F2CMPE, is proposed to produce reliable entropy values based on F2C signals which contain low and high-frequency information. Moreover, the $\mathrm{F} 2 \mathrm{C}$ procedure can reduce the influence of decreased data length in temporal time series at large scale factors caused by the coarse-grained process.

- An experimental study is carried out to investigate the performance of F2CMPE and MPE for bearing fault diagnosis based on Support Vector Machine (SVM) under 
TABLE I: Comparison of related work for bearing fault diagnosis based on MPE and PE.

\begin{tabular}{llllll} 
& Wu et al. [8] & Tiwari et al. [9] & Zhao et al. [10] & Vakharia et al. [11] & Zhang et al. [12] \\
\hline \hline Year & 2012 & 2015 & 2015 & 2015 \\
\hline Objects considered & $\begin{array}{l}\text { Four bearing } \\
\text { conditions }{ }^{1}\end{array}$ & $\begin{array}{l}\text { Four bearing } \\
\text { conditions }\end{array}$ & $\begin{array}{l}\text { Four bearing } \\
\text { conditions }\end{array}$ & $\begin{array}{l}\text { Four bearing } \\
\text { conditions under four } \\
\text { different loads }\end{array}$ \\
\hline $\begin{array}{l}\text { Signal processing } \\
\text { method }\end{array}$ & N/A & N/A & $\begin{array}{l}\text { Wavelet packet } \\
\text { decomposition }\end{array}$ & $\begin{array}{l}\text { MPE-based continuous } \\
\text { wavelet transform }\end{array}$ & $\begin{array}{l}\text { Ensemble empirical } \\
\text { mode decomposition }\end{array}$ \\
\hline $\begin{array}{l}\text { Feature extraction } \\
\text { applied }\end{array}$ & MPE & MPE & $\begin{array}{l}\text { MPE of each } \\
\text { coefficient }\end{array}$ & $\begin{array}{l}\text { Ten time-domain } \\
\text { features }\end{array}$ & PE of the first 5 IMFs \\
\hline Classifiers applied & SVM & ANFIS & $\begin{array}{l}\text { Hidden Markov } \\
\text { model }\end{array}$ & ANN and SVM & Optimized SVM \\
\hline
\end{tabular}

${ }^{1}$ Four bearing conditions: one healthy condition, and three fault conditions: damages on inner race, outer race, and rolling element.

different bearing conditions and fault severity levels.

The rest of this paper is organized as follows: Section II reviewed related work based on MPE and PE with a multiplescale method. Section III presents the proposed F2CMPE method and F2C procedure based on WPD and PE. Section IV shows the experimental investigation of the proposed method and gives comparative results in contrast with that of the MPE. Finally, conclusions are drawn in Section V.

\section{RELATED WORK}

Based on PE and the coarse-grained procedure in MSE, MPE was later developed by Azia and Arif [4], [5]. In contrast with SE used in MSE, PE enjoys the advantages of fast calculation and invariance for non-linear monotonous transformations [1]. Similar to MSE, the calculation of MPE mainly includes two procedures:

1) Firstly, given a time series, $x=x_{1}, x_{2}, \cdots, x_{N}$, a set of coarse-grained time series can be obtained over different scale factors $\tau$ according to Eq. (1).

$$
y_{j}^{(\tau)}=\frac{1}{\tau} \sum_{i=(j-1) \tau+1}^{j \tau} x_{i}, 1 \leq j \leq \frac{N}{\tau}, N>\tau
$$

2) Afterwards, the complexity in each coarse-grained time series is calculated by using normalized PE values [1].

$$
P E=-\ln (m !)^{-1} \sum_{j=1}^{m !} p\left(\pi_{j}\right) \ln \left(p\left(\pi_{j}\right)\right)
$$

where for each permutation, $\pi_{j}$, the relative frequency can be determined by:

$$
p\left(\pi_{j}\right)=\frac{\text { Number }\left\{X_{i}^{m}\right\}}{N-(m-1) \lambda}
$$

where Number $\left\{X_{i}^{m}\right\}$ represents the number of $X_{i}^{m}$ which is consistent with the type $\pi, m$ is the embedding dimension, and $\lambda$ is the time delay in the calculation of PE. For brevity, more details about PE and MPE can be found in [1], [4], [5].

After MPE method was put forward, it was adopted in fault diagnosis of bearings by offering the complexity over different scales based on PE, as reviewed in Table I. For instance, Wu et al. [8] investigated the feasibility and performance of MPE for bearing fault diagnosis, in which MPE was used for feature extraction from bearing vibration signals. After that, SVM was applied to classify different bearing conditions. It was reported that MPE could extract the features with high distinguishably. Similarly, Tiwari [9] used MPE and an adaptive neuro-fuzzy classifier for bearing diagnosis, in which the former was applied for fault symptom extraction, and the later was applied for fault identification and classification. Meanwhile, the comparative studies showed that the performance of MPE outperformed statistical features (e.g., kurtosis, skewness, mean, and standard deviation) when the same classifier was used for fault prediction. Furthermore, Zhao [10] developed a method based on WPD and MPE, in which vibration signals were first decomposed to subfrequency coefficients and the average values of MPE for each sub-frequency band were then calculated. The hidden Markov model was finally used to identify the fault patterns of rolling bearing.

Additionally, MPE was applied as a tool to select the best wavelet kernel in continuous wavelet transform [11]. The mother wavelet having the minimum permutation entropy (in their method the PE lacks of a negative sign) was finally chosen to extract time and frequency information for feature extraction by using time-domain features. Later, Artificial Neural Network (ANN) and SVM were respectively applied to recognize bearing conditions for a comparative purpose. It showed that the mother wavelet used in wavelet analysis having minimum permutation could achieve a positive classification accuracy in bearing fault diagnosis. Besides, PE was also applied as a feature extraction role in [12], in which signals were firstly decomposed to Intrinsic Mode Functions (IMFs) using ensemble empirical mode decomposition. After that, PE was applied to estimate the complexity in the first 5 IMFs, the values of which were finally fed into a classifier for fault classification. As can be concluded, MPE and PE have been popularly applying for discriminating the change of complexity in bearing vibration signals. Indeed, the way how to extract the dynamic change hidden in low or high- 


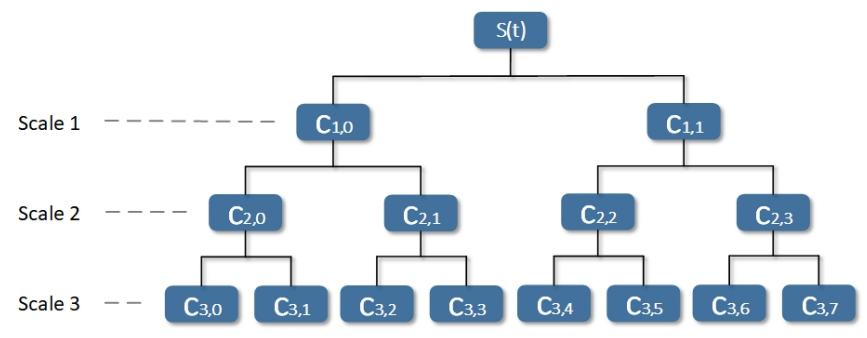

Fig. 1: An example of a three-level WPD tree.

frequency components significantly impacts the performance of characterizing fault symptoms from the original signal using entropy measure. Therefore, in this study, the proposed measure, named F2CMPE, is developed and presented to provide more accurate and reliable multiple-scale PE measurements.

\section{Fine-to-CoARse Multiscale Permutation ENTROPY(F2CMPE)}

This section introduces the proposed F2CMPE method and F2C procedure based on WPD and PE. Herein, WPD is first applied to generate $\mathrm{F} 2 \mathrm{C}$ signals by reconstructing selected wavelet coefficients. WPD is essentially a multi-resolution analysis that can provide the good time and frequency resolution as one of powerful wavelet analysis methods [13]. Similar to discrete wavelet decomposition, WPD is also a filtering operation that decomposes a signal into approximation parts (low-pass filter) and detail parts (high-pass filter) by iteratively using wavelet filtering operation until desired frequency resolution is achieved [14]. Hence, given a $j^{\text {th }}$ decomposition level, $2^{j}$ sets of wavelet coefficients can be obtained, and they nearly spilled the whole frequency spectrum to $2^{j}$ subfrequency bands. A wavelet packet function $\omega_{j, t}^{\theta}(t)$ is defined as [15]:

$$
\omega_{j, k}^{\theta}(t)=2^{j / 2} \omega_{0,0}^{\theta}\left(2^{j} t-k\right)
$$

where $j$ and $k$ are the scaling (frequency localization) parameter and the translation (time localization) parameter respectively; $\theta=0,1,2, \ldots$ is the oscillation parameter. Therefore, $2^{j}$ sets of wavelet packet coefficients of the signal $x(t)$ are obtained as follows:

$$
C_{j, n}=\left\{\int_{-\infty}^{\infty} x(t) \omega_{j, k}^{n}(t) d t, \mid 1 \leq k \leq \frac{N}{2^{j}}\right\}
$$

where $j$ is the decomposition level, $N$ is the data length of the original signal, and $n\left(0 \leq n \leq 2^{j}-1\right)$ is the order of wavelet coefficient in a given $j^{t h}$ decomposition level obtained in a wavelet tree. From each vector $C_{j, n}$, a reconstruction signal $R_{j, n}$ of length $N$ can be produced, by setting the coefficients of all the other vectors in level $j$ to zero and implementing the wavelet packet tree in the inverse procedure. Hence, the frequency range of $R_{j, n}$ is $F_{j} \approx F_{s} / 2^{j}$, where $F_{s}$ is the sampling frequency. The procedure of WPD in level three is illustrated in Fig. 1. Therefore, based on the advantages of WPD, the F2CMPE algorithm is described as follows:

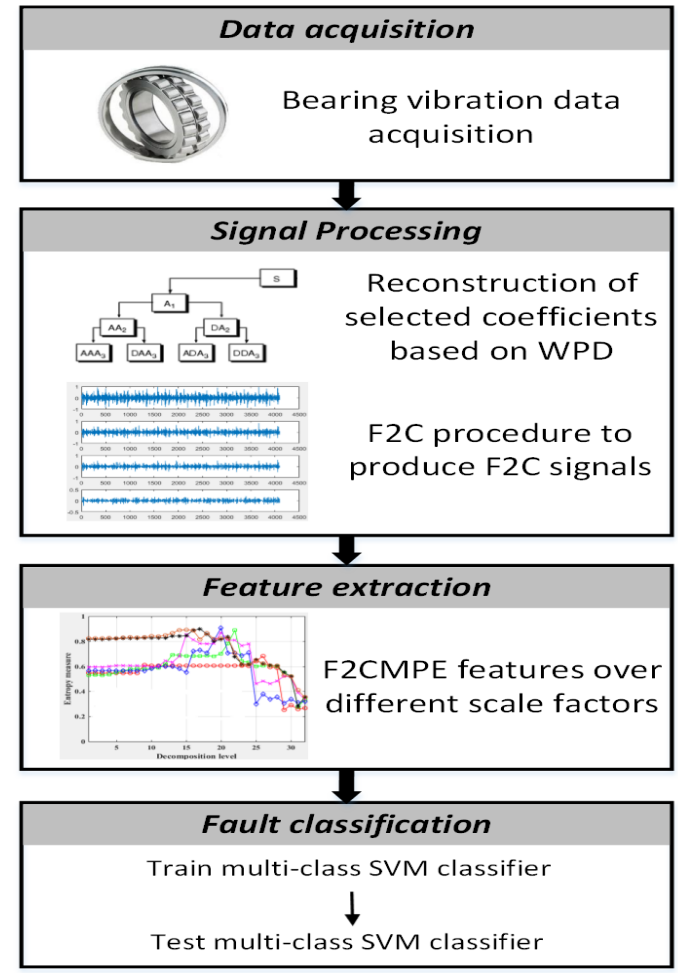

Fig. 2: Framework of the proposed bearing fault diagnosis method based on the F2CMPE and SVM.

1) Firstly, apply WPD to decompose the original signal to $1^{\text {st }}$ level, then only select the approximate coefficient $C_{1,0}$ for further decomposition;

2) Continue to use WPD to decompose the approximate coefficient, $C_{1,0}$ obtained from step 1 , to a given $j^{\text {th }}$ level; therefore, $2^{j}$ number of wavelet coefficients $C_{j, n}$ can be obtained. Then, reconstruct wavelet packet coefficients in the $j^{\text {th }}$ level to the signals with length $N$, $R_{j, n},\left(0 \leq n \leq 2^{j}-1\right)$;

3) F2C procedure: construct F2C signals by successively removing the reconstruction signals having subfrequency bands, starting from the accumulation of reconstruction signals in the $j^{\text {th }}$ level:

$S_{k}=\sum_{i=0}^{2^{j}-k} R_{j, i}, 0 \leq i \leq 2^{j}-1,1 \leq k \leq 2^{j} ;$

4) Perform PE to estimate the complexity of F2C signals $\left(S_{k}, 1 \leq k \leq 2^{j}\right)$ over different scales. Herein, the number of F2CMPE scale factors is equal to $2^{j}$ wavelet nodes obtained from the $j^{\text {th }}$ decomposition level in step 2.

Hence, a new bearing fault diagnosis method can be developed based on the proposed F2CMPE and SVM used as feature extraction and fault classification methods respectively, the flowchart of which is presented in Fig. 2. In this study, the approximate coefficient in the first decomposition level obtained from the original signal is further used for constructing F2C signals. Indeed, the coarse-grained signal at 2-th scale in MPE also has the half frequency spectrum of the original 


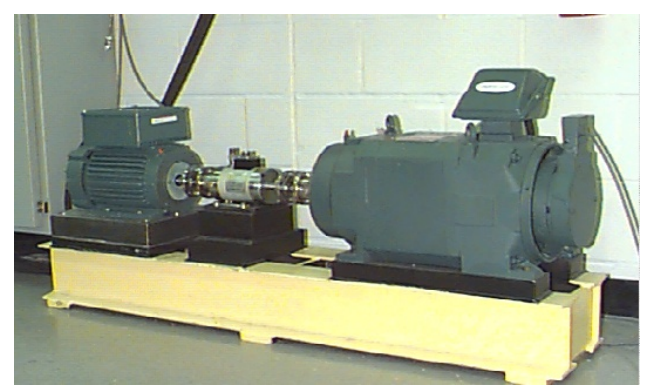

Fig. 3: CWRU bearing testbed [16].

signal. Furthermore, by reconstructing wavelet coefficients to signals having the same data length with that of the original signal, the effect of decreased data length in MPE can be reduced, and the efficiency is finally improved. Besides, the F2CMPE not only considers low-frequency but also highfrequency information by means of the decomposition capability of WPD analysis in extracting good time and frequency information from non-stationary signals. Moreover, it should be noticed that our proposed method is different with [10], in which MPE was directly used to estimate the complexity of each wavelet coefficient. In contrast, our proposed method applies PE to calculate entropy information from F2C signals produced by using reconstruction signals from WPD analysis and the $\mathrm{F} 2 \mathrm{C}$ procedure.

\section{EXPERIMENTAL STUDY}

This section presents an experimental study to investigate and give a comparative performance of the F2CMPE and MPE methods for bearing fault diagnosis based on SVM.

\section{A. Experiment Setup}

Experimental data with six bearing states and two kinds of fault severity levels were analyzed to investigate the feasibility and effectiveness of the proposed method. The experimental data were obtained from the Case Western Reserve University Bearing Data Center [16]. The experiment system is illustrated in Fig. 3. The vibration signals of bearing were collected under four conditions of rolling bearing, i.e., the normal condition (Norm), and the damage on the inner race (IR), the outer race (OR) at 6 o'clock, and the ball element (BE). In this study, for all fault conditions, two defect sizes of point fault were considered $0.1778 \mathrm{~mm}$ and $0.5334 \mathrm{~mm}$ and depth $0.2794 \mathrm{~mm}$, respectively. For each state, there are in total 29 samples (4, 096 data points in each sample) under motor load 3, the $1730 \mathrm{r} / \mathrm{min}$ and sampling frequency $12 \mathrm{kHz}$. The detail specification of each state of a bearing is presented in Table II.

\section{B. Experimental Results}

It is noticed that it is difficult to distinguish these six states of rolling bearing by observing time-domain waveforms as shown in Fig. 4. In the following, our proposed method F2CMPE and MPE are used to investigate and give the comparative performance of bearing fault diagnosis using the experimental data sets. There are in total 29 samples for each
TABLE II: Description of bearing states and class labels.

\begin{tabular}{lllll} 
States & $\begin{array}{l}\text { Fault } \\
\text { diameter }\end{array}$ & $\begin{array}{l}\text { Class } \\
\text { label }\end{array}$ & $\begin{array}{l}\text { Number of } \\
\text { training samples }\end{array}$ & $\begin{array}{l}\text { Number of } \\
\text { testing samples }\end{array}$ \\
\hline \hline IR1 & 0.1778 & 1 & 14 & 15 \\
\hline IR2 & 0.5334 & 2 & 14 & 15 \\
\hline OR1 & 0.1778 & 3 & 14 & 15 \\
\hline OR2 & 0.5334 & 4 & 14 & 15 \\
\hline BE & 0.1778 & 5 & 14 & 15 \\
\hline Norm & 0 & 6 & 14 & 15 \\
\hline
\end{tabular}

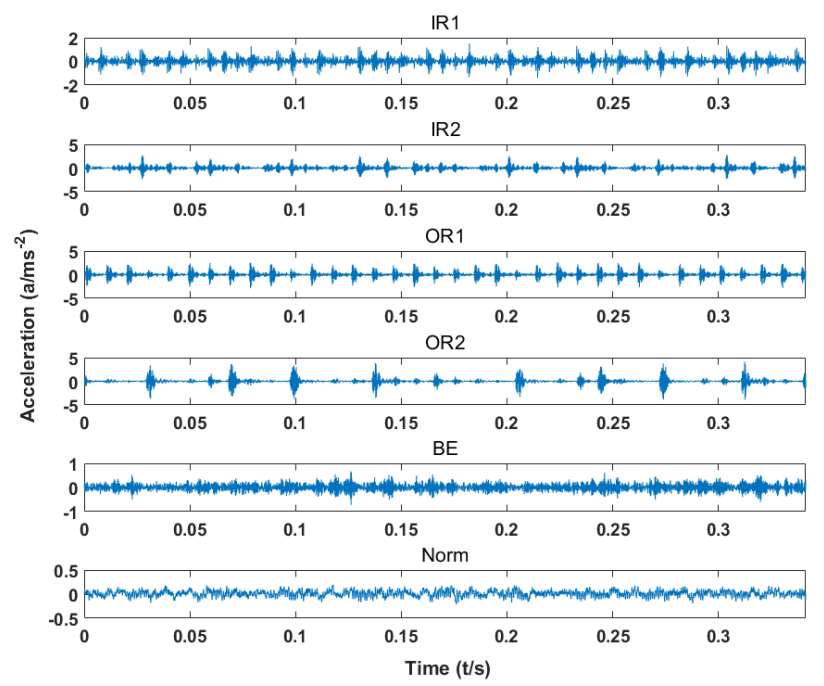

Fig. 4: Time-domain waveforms of rolling bearing vibration signals with six conditions.

condition; therefore, totally 174 samples are then obtained. Firstly, the F2CMPE and MPE algorithms are used to extract features from the original vibration signals of rolling bearing over different scales. Later, the mean and standard deviation indicators of the F2CMPE and MPE features in each class are obtained, as illustrated in Fig. 5. Particularly, in the calculation of F2CMPE features, 'db4' mother wavelet is used in WPD analysis for decomposing signals and reconstructing wavelet coefficients. After that, the $\mathrm{F} 2 \mathrm{C}$ procedure is used to produce F2C signals. Then, in the calculation of PE, the embedding dimension $m$ is set to 5 and the time delay $\lambda$ is set to 3 according to suggested parameters given by [1].

Besides, the performance of F2CMPE and MPE features with 16 and 32 scales is comparatively studied. That is, features with different scales are first extracted from the original vibration signal, and later the varying number of the F2CMPE and MPE features, considered as feature vectors, are then fed into SVM for fault classification. As can be seen from Fig. 5 (a) and (b), the entropy measurements obtained from the F2CMPE initially keep steady and then gradually decrease to roughly $0.3-0.4$. This can be explained that $\mathrm{F} 2 \mathrm{C}$ signals at low scales contain both high and lowfrequency information; therefore, with a larger scale, the high- 


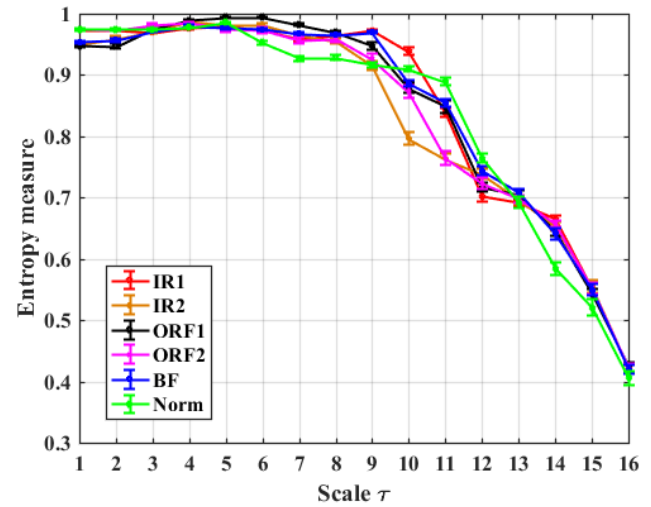

(a) F2CMPE features of vibration signals over 16 scales

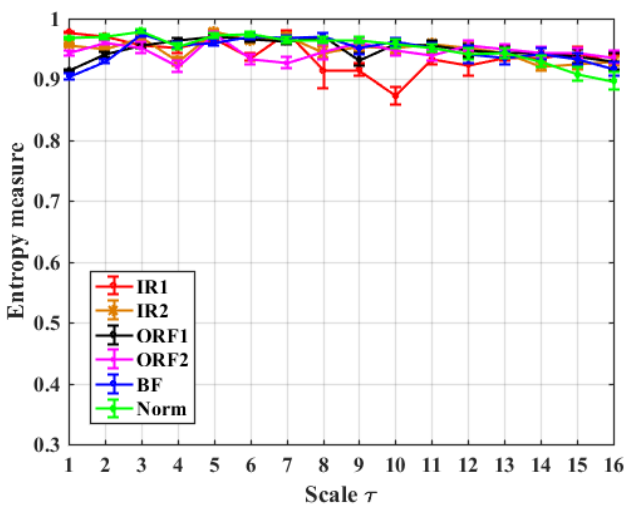

(c) MPE features of vibration signals over 16 scales

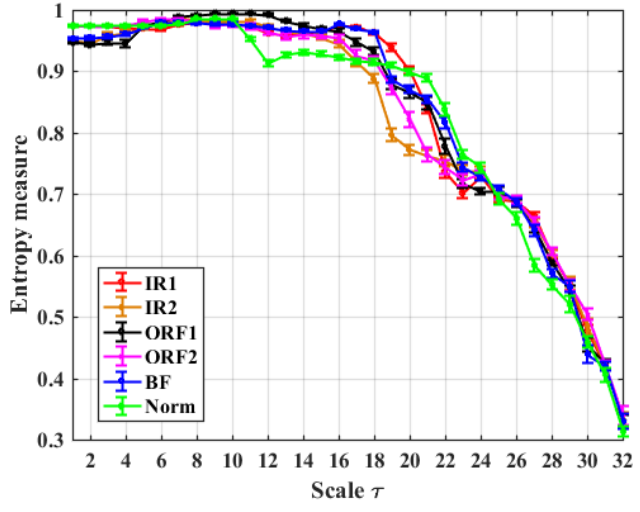

(b) F2CMPE features of vibration signals over 32 scales

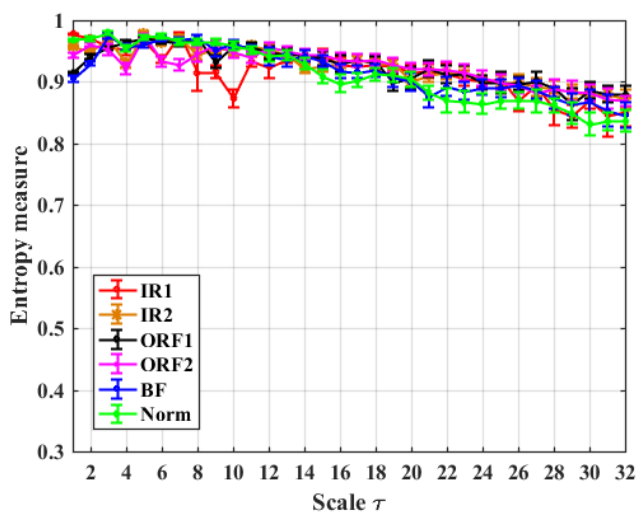

(d) MPE features of vibration signals over 32 scales

Fig. 5: The entropy results of bearing vibration signals based on the F2CMPE and MPE over 16 and 32 scales respectively.

frequency information is consecutively removed, the result of which finally decreases the complexity of F2C signals at large scales where low-frequency remains. Besides, it can be noticed that bearing fault symptoms could be distinguished in low-frequency components, namely in F2C signals with large scales containing low-frequency components. Entropy measure in Fig. 5 (a) and (b) indicates that six bearing conditions can be relatively distinguished, especially between $9-12$ scales in $4^{\text {th }}$ decomposition level and $18-23$ scales in $5^{\text {th }}$ decomposition level respectively. Furthermore, the F2CMPE measure gradually decreases along with the increasing scale factor, the reason of which is that $\mathrm{F} 2 \mathrm{C}$ signals at large scales only have low-frequency information and become more and more smooth; therefore, the complexity accordingly decreases. Besides, by comparing the F2CMPE under 16 scales and 32 scales in Fig. 5 (a) and (b) respectively, it can be easily found that the energy change in medium scales in $5^{\text {th }}$ level is more obvious than that in $4^{\text {th }}$ level.

Comparably, PE measurements obtained from the MPE keep steady from the starting to the end. The entropy values at large scales are slightly smaller than that of small scales. Moreover, ones can hardly distinguish the trends of six conditions of bearing from MPE curves. Indeed, only low-frequency information is extracted in the MPE; therefore, with an increasing scale, coarse-grained time series becomes more and more smooth, and decreased data length may also produce inappropriate entropy values in the calculation of PE. As a result, the coarsegrained procedure may yield imprecise entropy measure for estimating the dynamic changes of bearing vibration signals.

For comparison, without applying any feature selection methods, the feature vectors, consisted of different number of F2CMPE and MPE features at different scales, are fed into SVM for fault classification. The identification accuracy rate of diagnosis methods based on varying number of F2CMPE and MPE features, and SVM classifier is shown in Fig. 6. In this study, LIBSVM Matlab Toolbox is applied for data training and testing using SVM [17]. A grid search method is applied to search best parameters, and a 9-fold cross-validation method is used for the validation of the proposed rotating shaft fault diagnostic approach. The Fig. 6 indicates that MPE can achieve a better accuracy rate when the first six features are applied for fault classification. Meanwhile, the F2CMPE method rapidly catches up and increases to $100 \%$ when the first seven features were applied. ones can find that our proposed method can continuously maintain $100 \%$ accuracy rate by using more than eight features. However, the accuracy rate of MPE cannot give a stable performance, especially when more features at large scales are used. Moreover, this phenomenon can be more severe as can be seen in Fig. (b), in which the accuracy rate relatively fluctuates between $96 \%$ 


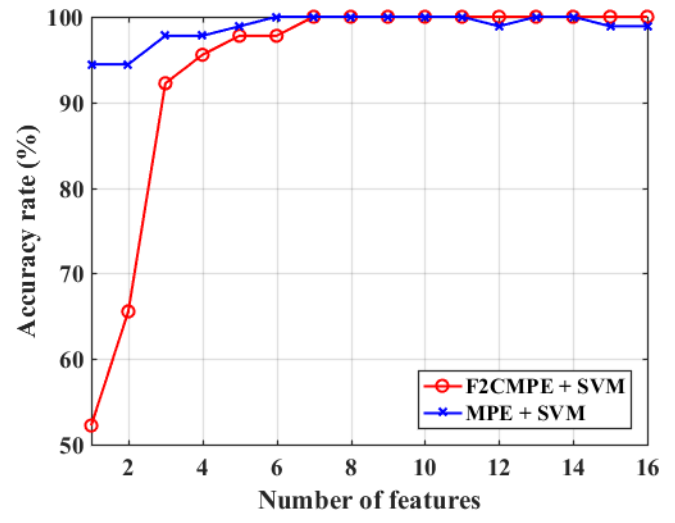

(a) Comparison of accuracy rate over 16 scales

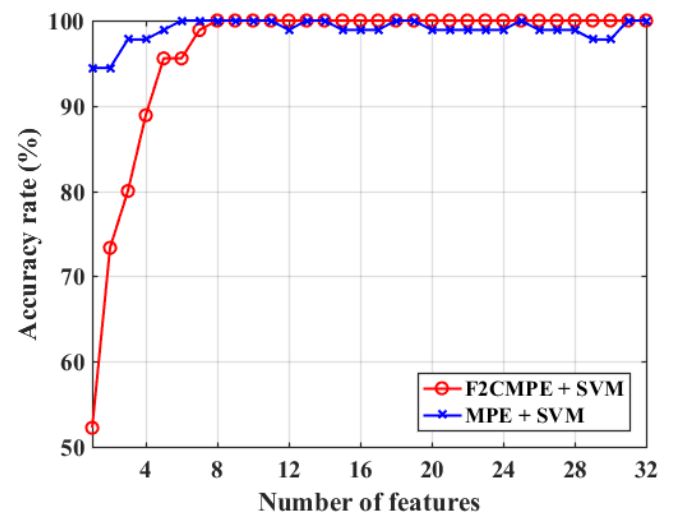

(b) Comparison of accuracy rate over 32 scales

Fig. 6: Comparative accuracy rate for bearing diagnosis using different number of F2CMPE and MPE features based on SVM.

and $100 \%$ when MPE features at larger scales are increasingly added in fault feature vectors. To sum up, in this study, the proposed method using F2CMPE for bearing fault diagnosis can give consistent entropy values and stable positive accuracy rate in contrast with that of the MPE. Experimental results indicate that the F2CMPE algorithm can efficiently detect and identify bearing failures with different states.

\section{CONCLUSIONS}

In this paper, a new non-linear entropy method, named F2CMPE, is presented for identifying incipient faults in rolling bearings. Experimental analysis was conducted to investigate the performance of the F2CMPE and MPE used for discriminating bearing conditions with different severity levels. Results demonstrated that F2CMPE can obtain coherent fault features by characterizing the dynamic change of bearing vibration signals. Besides, experimental results also showed that the bearing diagnosis method based on the F2CMPE and SVM could provide a positive and stable fault classification accuracy rate in contrast with that based on MPE, which indicates the feasibility and effectiveness of the proposed method for fault diagnosis of rolling bearing.

\section{ACKNOWLEDGEMENT}

This work is partially supported by International and Hong Kong, Macao \& Taiwan collaborative innovation platform and major international cooperation projects of colleges in Guangdong Province (No.2015KGJHZ026), and The Natural Science Foundation of Guangdong Province (No.2016A030307029).

\section{REFERENCES}

[1] R. Yan, Y. Liu, and R. X. Gao, "Permutation entropy: a nonlinear statistical measure for status characterization of rotary machines," Mechanical Systems and Signal Processing, vol. 29, pp. 474-484, 2012.

[2] Z. Huo, Y. Zhang, L. Shu et al., "A short survey on fault diagnosis of rotating machinery using entropy techniques." 3rd EAI International Conference on Industrial Networks and Intelligent Systems, 2017.

[3] A. Humeau-Heurtier, "The multiscale entropy algorithm and its variants: a review," Entropy, vol. 17, no. 5, pp. 3110-3123, 2015.

[4] M. Costa, A. L. Goldberger, and C.-K. Peng, "Multiscale entropy analysis of complex physiologic time series," Physical review letters, vol. 89, no. 6, p. 068102, 2002.
[5] W. Aziz and M. Arif, "Multiscale permutation entropy of physiological time series," in 9th International Multitopic Conference, IEEE INMIC 2005. IEEE, 2005, pp. 1-6.

[6] J. F. Valencia, A. Porta, M. Vallverdu, F. Claria, R. Baranowski, E. Orlowska-Baranowska, and P. Caminal, "Refined multiscale entropy: Application to 24-h holter recordings of heart period variability in healthy and aortic stenosis subjects," IEEE Transactions on Biomedical Engineering, vol. 56, no. 9, pp. 2202-2213, 2009.

[7] S.-D. Wu, C.-W. Wu, S.-G. Lin, C.-C. Wang, and K.-Y. Lee, "Time series analysis using composite multiscale entropy," Entropy, vol. 15, no. 3, pp. 1069-1084, 2013.

[8] S.-D. Wu, P.-H. Wu, C.-W. Wu, J.-J. Ding, and C.-C. Wang, "Bearing fault diagnosis based on multiscale permutation entropy and support vector machine," Entropy, vol. 14, no. 8, pp. 1343-1356, 2012.

[9] R. Tiwari, V. K. Gupta, and P. Kankar, "Bearing fault diagnosis based on multi-scale permutation entropy and adaptive neuro fuzzy classifier," Journal of Vibration and Control, vol. 21, no. 3, pp. 461-467, 2015.

[10] L.-Y. Zhao, L. Wang, and R.-Q. Yan, "Rolling bearing fault diagnosis based on wavelet packet decomposition and multi-scale permutation entropy," Entropy, vol. 17, no. 9, pp. 6447-6461, 2015.

[11] V. Vakharia, V. Gupta, and P. Kankar, "A multiscale permutation entropy based approach to select wavelet for fault diagnosis of ball bearings," Journal of Vibration and Control, vol. 21, no. 16, pp. 3123-3131, 2015.

[12] X. Zhang, Y. Liang, J. Zhou et al., "A novel bearing fault diagnosis model integrated permutation entropy, ensemble empirical mode decomposition and optimized SVM," Measurement, vol. 69, pp. 164-179, 2015.

[13] Z. Huo, Y. Zhang, P. Francq, L. Shu, and J. Huang, "Incipient fault diagnosis of roller bearing using optimized wavelet transform based multi-speed vibration signatures," IEEE Access, 2017.

[14] S. G. Mallat, "A theory for multiresolution signal decomposition: the wavelet representation," IEEE transactions on pattern analysis and machine intelligence, vol. 11, no. 7, pp. 674-693, 1989.

[15] T. Ogden, Essential wavelets for statistical applications and data analysis. Springer Science \& Business Media, 2012.

[16] "Case Western Reserve University Bearing Data Center," (Date last accessed 20-April-2018). [Online]. Available: http://csegroups.case.edu/ bearingdatacenter/home

[17] "LIBSVM Matlab Toolbox," (Date last accessed 20-April-2018). [Online]. Available: https://www.csie.ntu.edu.tw/ cjlin/libsvm/

\section{ABBREVIATIONS}

F2CMPE Fine-to-Coarse Multisccale Permutation Entropy

MPE Multiscale Permutation Entropy

MSE Multiscale Entropy

PE Permutation Entropy

SE Sample Entropy

SVM Support Vector Machine

WPD Wavelet Packet Decomposition 